\title{
Pituitary Apoplexy Leading to Cerebral Infarction: A Systematic Review
}

\author{
Qiuhong Jiang $^{\mathrm{a}}$ Su Xiao ${ }^{\mathrm{a}}$ Liming Shu $^{\mathrm{b}}$ Xinyi Huang ${ }^{\mathrm{b}}$ Xiuhui Chen $^{\mathrm{a}}$ \\ Hua Hong ${ }^{\text {a }}$ \\ aDepartment of Neurology, The First Affiliated Hospital, Sun Yat-sen University, Guangdong Provincial Key \\ Laboratory of Diagnosis and Treatment of Major Neurological Diseases, National Key Clinical Department and Key \\ Discipline of Neurology, Guangzhou, China; 'bepartment of Neurology, The Seventh Affiliated Hospital, Sun Yat- \\ sen University, Shenzhen, China
}

\section{Keywords}

Pituitary apoplexy · Cerebral infarction · Prognosis

\begin{abstract}
Background: Cerebral infarction caused by pituitary apoplexy (PA) is rare. To characterize the clinical features of cerebral infarction caused by PA, we performed a systematic review. Summary: The clinical symptoms are mainly sudden headache, hemiplegia, visual impairment, disturbance of consciousness, and ophthalmalgia in patients with cerebral infarction caused by PA. Treatment for this type of infarction is different from treatment for general acute cerebral infarction. Compared to patients who underwent emergency surgery and conservative treatment, patients treated with delayed surgery showed a better prognosis and a lower mortality rate. Compared to patients who underwent craniotomy or conservative treatment, patients who underwent transsphenoidal surgery (TSS) not only improved well but also showed a lower mortality rate. Key Messages: PA rarely causes cerebral infarction, which is a critical condition with a poor prognosis and is more common in men. Delayed surgery and TSS appear to confer a better prognosis in patients with this condition.

(c) 2020 S. Karger AG, Basel
\end{abstract}

\section{Introduction}

Pituitary apoplexy (PA) manifests as acute pituitary infarction or haemorrhage, and the main symptoms are acute headache, impaired vision, and reduced level of consciousness $[1,2]$. PA was first reported by Bailey in 1989 , and the current incidence is approximately $0.6-$ $22 \%[1,3]$. However, acute cerebral infarction caused by PA involving adjacent blood vessels in the brain is rare. Although only a few cases have been reported, the morbidity and mortality of cerebral infarction caused by PA are more critical than those of general cerebral infarction; thus, this disease deserves our attention. This study aimed at improving the understanding of this rare but critical disease by reviewing cases and summarizing the clinical data and characteristics of cerebral infarction caused by PA in a systematic review.

Qiuhong Jiang and Su Xiao contributed equally to this article as first authors. karger@karger.com

(C) 2020 S. Karger AG, Basel

www.karger.com/ene

Karger ${ }^{\prime}=$
Prof. Hua Hong

Department of Neurology, The First Affiliated Hospital, Sun Yat-sen University

No. 58, Zhongshan Road 2, Yuexiu District

Guangzhou 510080 (China)

hhsums@126.com 
Fig. 1. Identified articles and the study selection process.

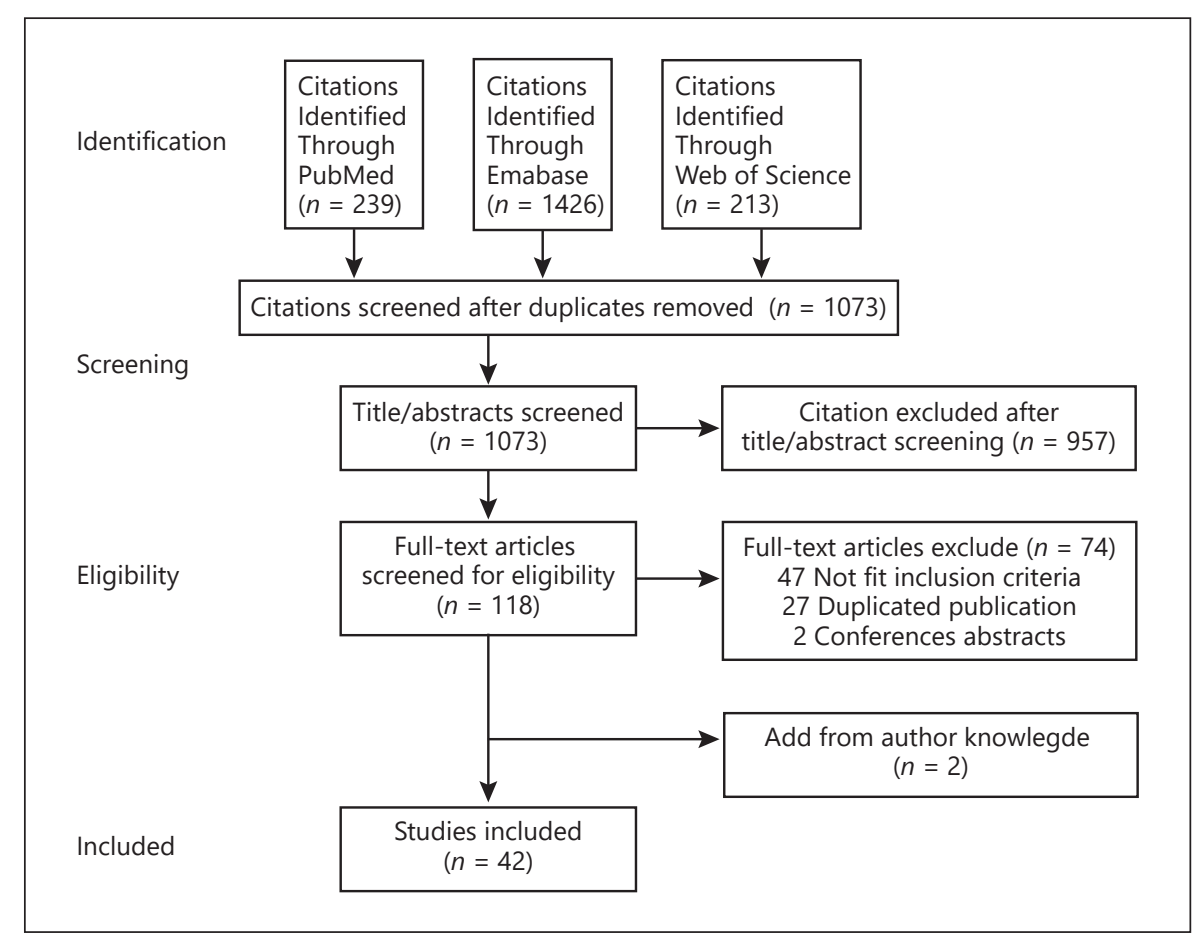

\section{Materials and Methods}

Document Retrieval

A literature search (from January 1950 to December 2018) on cerebral infarction caused by PA was performed using the reference databases PubMed, Web of Science, and Embase. The search keywords were as follows: PA, ischaemic stroke, cerebral infarction, cerebrovascular accident, vasospasm, and artery occlusion. Only defects with neurological symptoms plus imaging examination, such as CT or/and magnetic resonance imaging (MRI), digital subtraction angiography, or autopsy findings could be diagnosed as PA and cerebral infarction. The studies were assessed and the data were extracted independently by 2 assessors (J.Q.H. and X.S.), and divergences were unified through discussion. We applied the following criteria to screen articles: (1) diagnostic accuracy of PA leading to cerebral infarction, (2) a detailed description of the treatment process, and (3) records in English. We further excluded summaries of meetings or conferences and duplicate publications. Relevant data were extracted from the literature, including the first author, publication year, age and sex, mechanism of stroke, clinical symptoms, precipitating factors, affected blood vessels, treatment, prognosis, and pathological findings.

\section{Statistical Analysis}

We used SPSS 23.0 software. A descriptive analysis was conducted for each variable. Continuous normally distributed measurement data are expressed as $X \pm s$. Count data were evaluated with the $\chi^{2}$ test and are expressed as absolute values. Values $p<$ 0.05 were considered statistically significant.

\section{Results}

\section{Included Literature}

A total of 1,878 articles were retrieved (Fig. 1). After the selection process (Fig. 1), a total of 118 studies underwent full-text assessment. During screening, 74 articles did not conform to the inclusion criteria, and 2 articles were added according to the authors' knowledge. Finally, a total of 42 articles were chosen in the study, encompassing 46 patients, including 34 males and 12 females. The male-to-female ratio was $3: 1$. The mean age was 42.8 (range: 6-81) years. Before acute stroke onset, 4 patients were known to have a diagnosis of pituitary adenoma; the triggers for PA were determined in 10 patients and included head injury, angiography, fall, surgery for pituitary adenoma, delivery, leuprolide injection, triple bolus test, anticoagulative and antithrombotic therapy, and pituitary function test (Table 2).

\section{Clinical Symptoms}

The main clinical symptoms were sudden headache (71.70\%), visual impairment (47.8\%), and ophthalmalgia (26.1\%) caused by paralysis of the oculomotor nerve, trochlear nerve, and abducens nerve. Other symptoms included fever, dizziness, vomiting, photophobia, and memory deficit. The major neurological signs were hemiplegia (52.2\%), facial paralysis (10.9\%), and dysarthria 


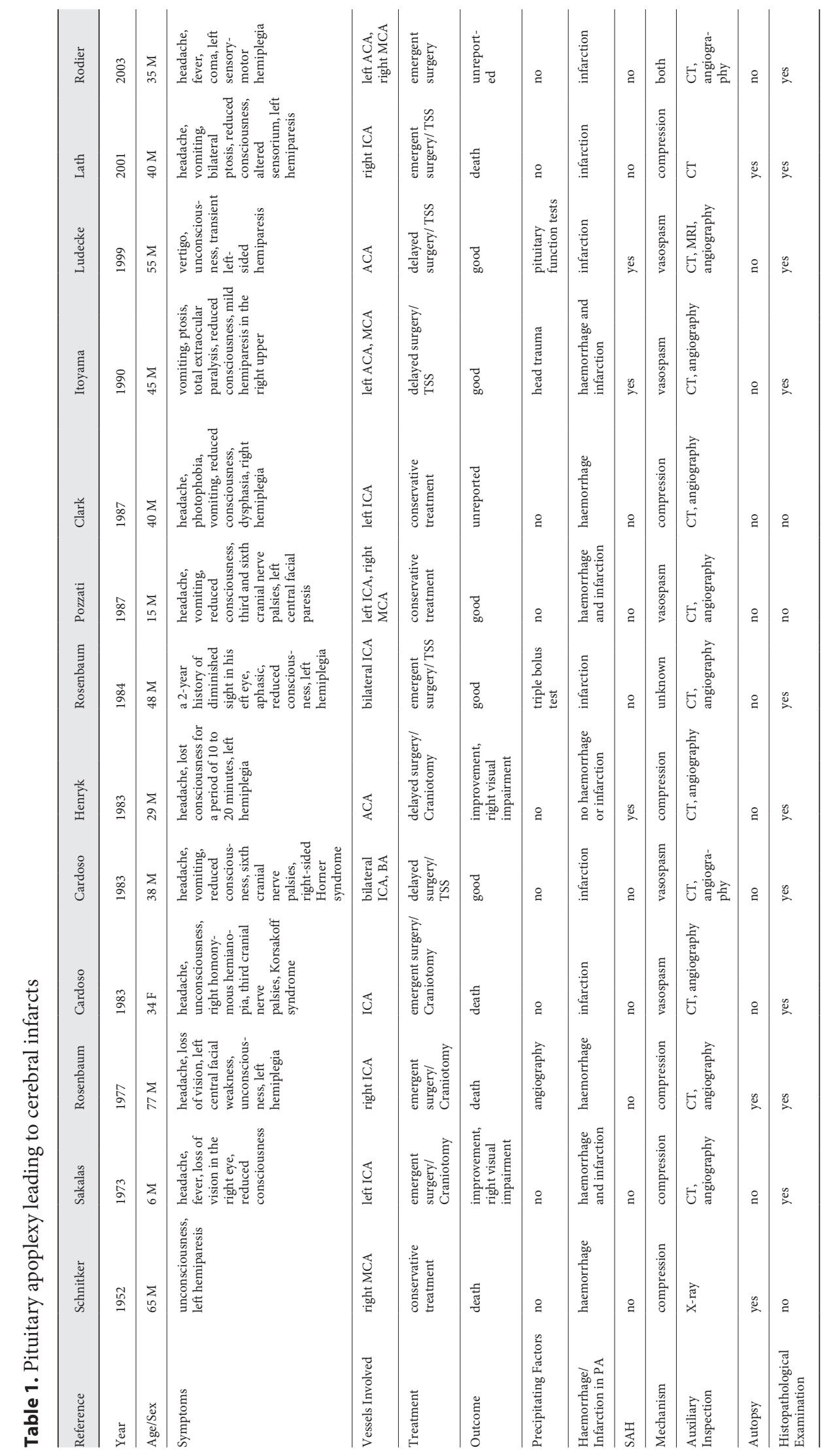




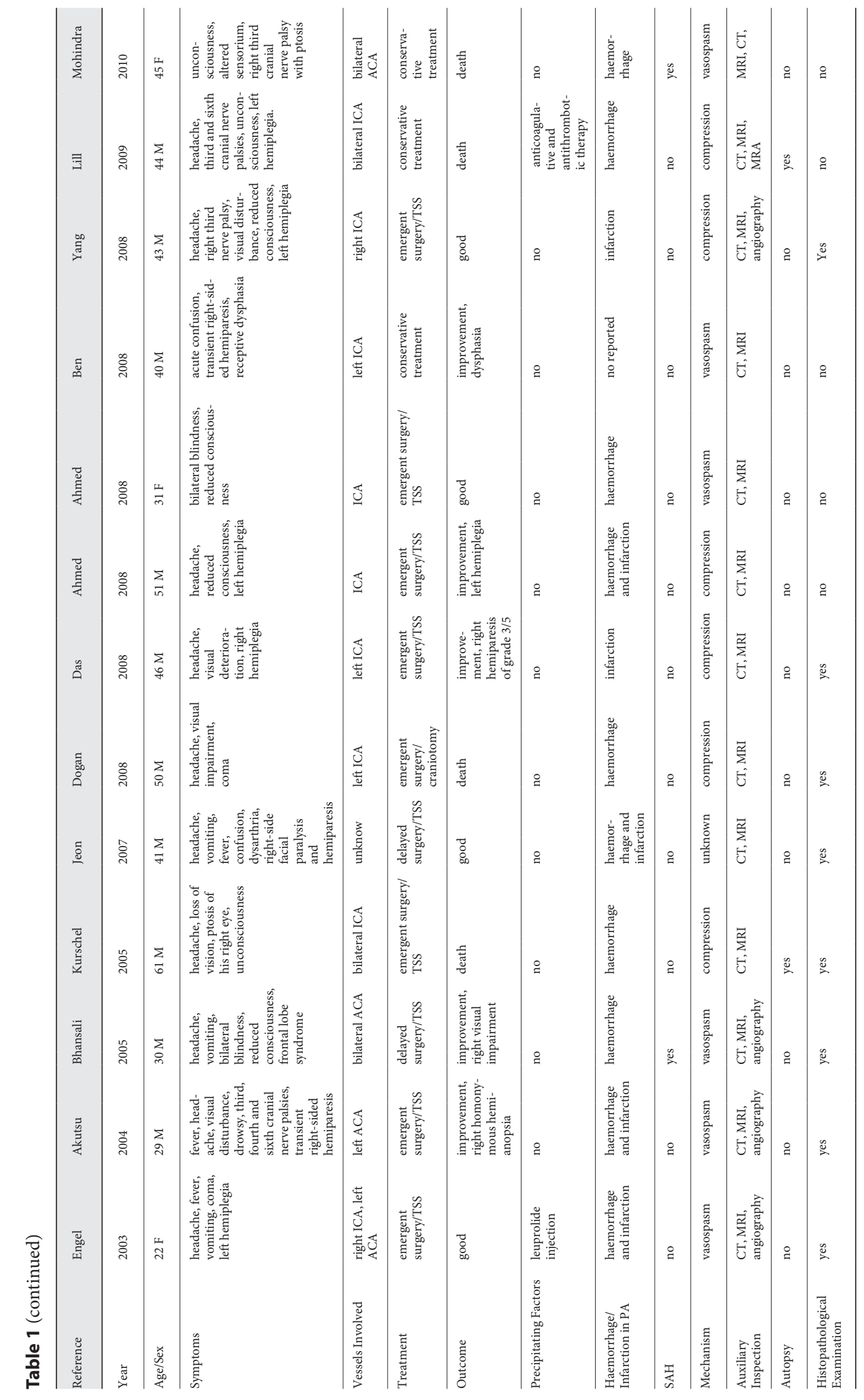




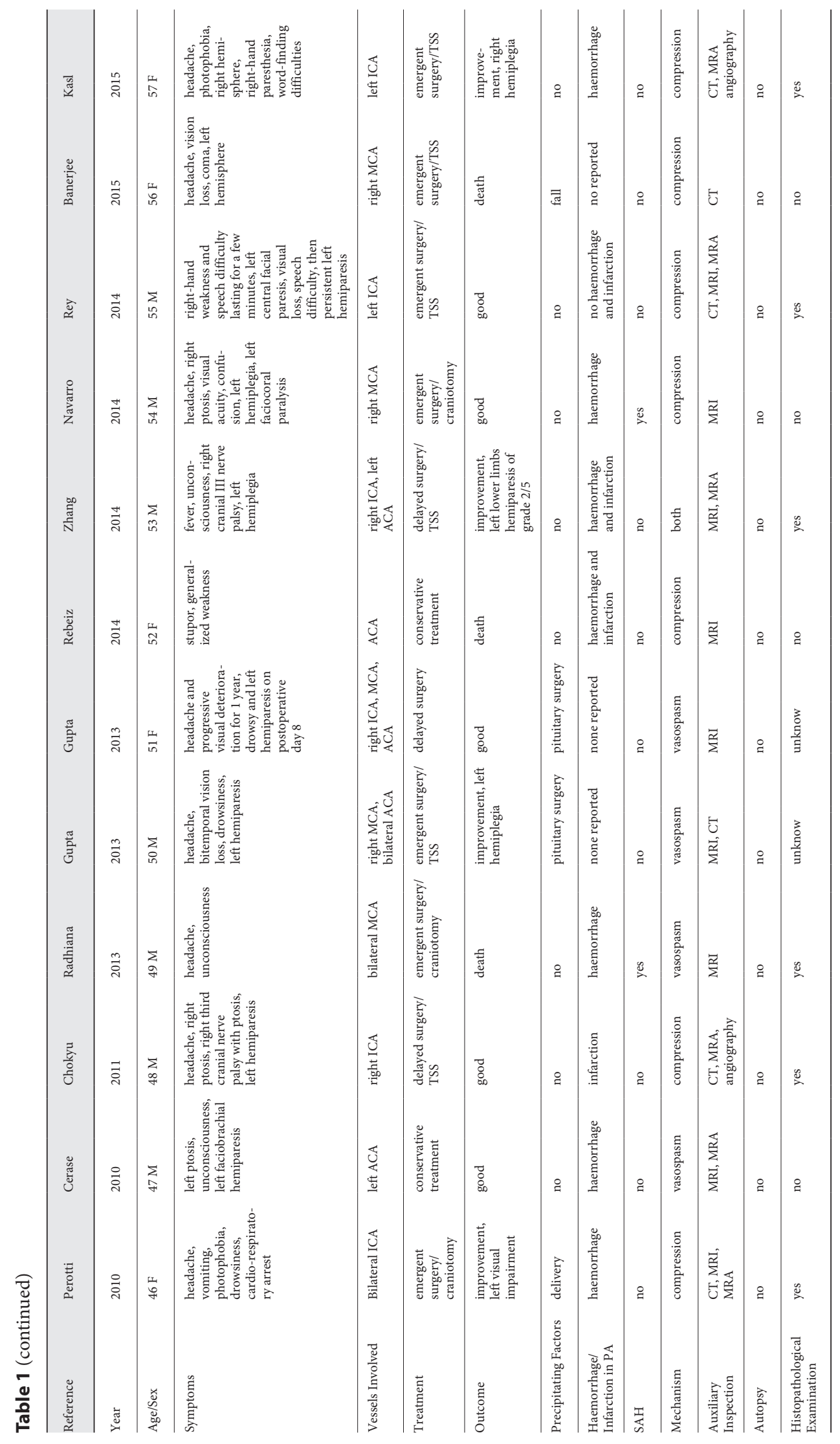




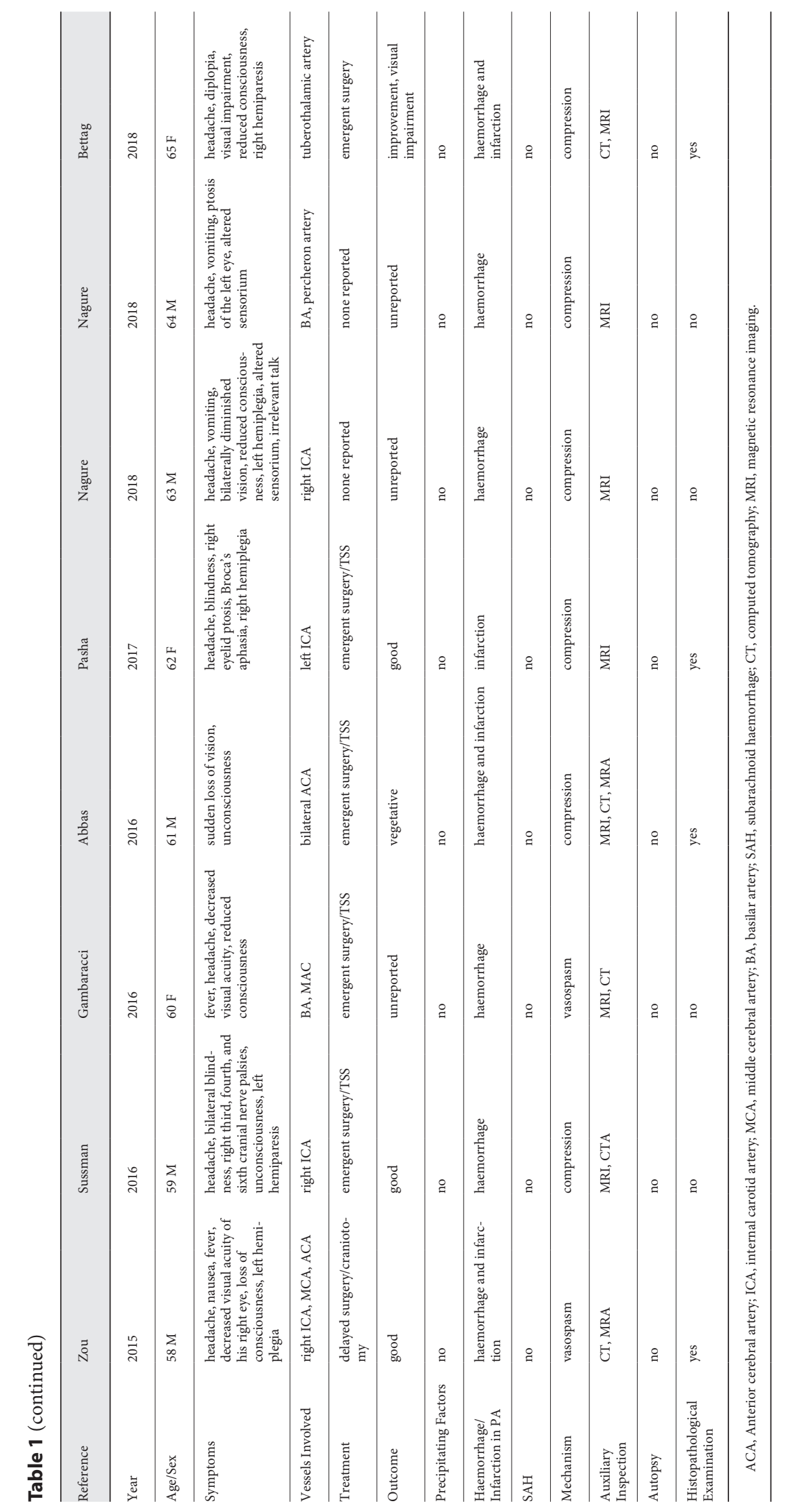


Table 2. Clinical features and risk factors of patients with PA leading to cerebral infarction

\begin{tabular}{lc}
\hline Demographic $(n=46)$ & $n(\%)$ \\
\hline Age, years, mean (range) & $42.8(6-81)$ \\
Sex, male & $34(73.9)$ \\
Pituitary adenoma history & $4(8.7)$ \\
Precipitating factors & $10(21.7)$ \\
Headache & $33(71.7)$ \\
Visual impairment & $22(47.8)$ \\
Ophthalmoplegia & $12(26.1)$ \\
Fever & $6(13.0)$ \\
Disturbed consciousness & $39(84.8)$ \\
Facial paralysis & $5(10.9)$ \\
Left/right hemiplegia & $24(52.2)$ \\
Sensory disturbance & $6(13.0)$ \\
Dysarthria/dysphasia & $6(13.0)$ \\
Korsakoff syndrome & $1(2.0)$ \\
Frontal lobe syndrome & $1(2.0)$ \\
Horner syndrome & $1(2.0)$ \\
SAH & $7(15.2)$ \\
Bilateral strokes & $13(28.3)$ \\
Watershed infarctions & $3(6.5)$ \\
CT & $35(76.1)$ \\
MRI & $32(69.6)$ \\
Angiography & $18(39.1)$ \\
Compression & $25(54.3)$ \\
Vasospasm & $17(40)$ \\
ICA & $27(58.7)$ \\
ACA & $15(32.6)$ \\
MCA & $11(24.0)$ \\
BA & $3(6.5)$ \\
\hline & \\
\hline &
\end{tabular}

$\mathrm{SAH}$, subarachnoid haemorrhage; $\mathrm{CT}$, computed tomography; MRI, magnetic resonance imaging; ICA, internal carotid artery; ACA, anterior cerebral artery; MCA, middle cerebral artery; BA, basilar artery.

(13\%). Thirty-nine patients developed disturbance of consciousness $(84.8 \%), 7$ patients $(15.2 \%)$ had subarachnoid haemorrhage, 13 patients $(27.3 \%)$ had bilateral stroke, and 3 patients $(6.5 \%)$ had watershed infarction (Table 2).

\section{Auxiliary Inspection}

CT scans were performed in 35 of 46 patients (76.1\%), MRI scans were performed in 32 patients (69.6\%), and angiography was performed in 18 patients (39.1\%). Twenty-eight patients underwent pathological examinations, and 6 patients underwent autopsy (Table 2).

\section{Mechanism of PA Leading to Cerebral Infarction}

Among the 46 patients, the mechanism was mechanical compression in 25 patients (54.3\%) and cerebral vaso-
Table 3. Treatment and outcome analysis of patients with stroke following PA $(n=41)$

\begin{tabular}{lrrll}
\hline \multirow{2}{*}{$\begin{array}{l}\text { Subgroup } \\
\text { n }\end{array}$} & $n$ & \multicolumn{2}{l}{ Outcome, $n(\%)$} & \\
\cline { 3 - 5 } & & good & improvement & death \\
\hline Emergent surgery & 24 & $8(33.3)$ & $9(37.5)$ & $7(29.2)$ \\
Delayed surgery & 10 & $7(70.0)$ & $3(30.0)$ & 0 \\
Conservative surgery & 7 & $2(28.6)$ & $1(14.3)$ & $4(57.1)$ \\
TSS & 23 & $12(52.2)$ & $7(32.4)$ & $4(17.4)^{\mathrm{a}}$ \\
Craniotomy & 9 & $2(22.2)$ & $3(33.3)$ & $4(44.4)$ \\
\hline
\end{tabular}

TSS, transsphenoidal surgery; PA, pituitary apoplexy. Good outcome indicates complete and nearly complete recovery. ${ }^{\text {a }}$ Including 1 patient who was in a vegetative state.

spasm in 17 patients (40\%) (Table 2). Two patients were considered to have involvement of both mechanisms, and the mechanism of cerebral infarction was unclear in $2 \mathrm{pa}$ tients. In 7 patients with subarachnoid haemorrhage, the mechanism was vasospasm in 5 and mechanical compression in 2 (Table 1 ).

\section{Affected Blood Vessels}

The main affected blood vessels were the internal carotid artery (ICA, 58.7\%), anterior cerebral artery (ACA, $32.6 \%$ ), middle cerebral artery (MCA, 25\%), and basilar artery (in 3 patients) (Table 2).

\section{Treatment and Outcomes}

No outcomes were reported for 5 patients (Table 1). For 41 patients, the relationship between treatment and prognosis was reported. Of these 41 patients, 11 (26.8\%) died, one was in a vegetative state, $17(41.5 \%)$ had a good prognosis, and $12(36.4 \%)$ with improvement had a certain degree of disability, namely, hemiplegia (5 patients), visual impairment (6 patients), and dysphasia (1 patient). Emergency surgery (within 7 days of presentation) was performed in 24 patients (58.5\%). Ten patients $(24.4 \%)$ underwent delayed surgery (at or more than 7 days after presentation; range: 7-67 days). Seven patients underwent conservative treatment, which mainly included hormone replacement therapy and symptomatic supportive care. In emergency surgery, 16 patients underwent transsphenoidal surgery (TSS), 7 patients with craniotomy, and one did not report the way of surgery. In delayed surgery, 7 patients underwent TSS, 2 patients with craniotomy, and one did not report the way of surgery (Table 1 ). Compared to patients who underwent emergency sur- 
gery and conservative treatment, patients treated with delayed surgery showed a better prognosis and a lower mortality rate ( 70 vs. $33.3 \%$ vs. $28.6 \%$; 0 vs. $29.2 \%$ vs. $57.1 \%$, $p<0.05$, respectively; Table 3$)$. Nine patients (21.9\%) underwent craniotomy and 23 (56.1\%) underwent TSS. Compared to patients who underwent craniotomy or conservative treatment, patients who underwent TSS not only improved well but also had a lower mortality rate (52.2 vs. $22.2 \%$ vs. $28.6 \%$; 17.4 vs. $44.4 \%$ vs. $57.1 \%, p<$ 0.05 , respectively; Table 3 ).

\section{Discussion}

PA leading to cerebral infarction is uncommon. Fortysix patients were included in this study [2,4-44]. A history of pituitary tumour was reported in $8.7 \%$ of patients, which is a very small proportion. Most cases of cerebral infarction following PA are sudden and may occur without any known trigger; however, some triggers have been proposed, including radiation therapy, bromocriptine therapy, foetation, head injury, anticoagulant therapy, diabetic ketoacidosis, and changes in intracranial cerebrospinal fluid pressure (caused by angiography, lumbar puncture, recurrent cough caused by respiratory tract infection, and general anaesthesia) and in the early postoperative period $[13,15,19]$. Of the patients collected in this study, $21.7 \%$ were identified as having predisposing factors for PA, such as angiography, head trauma, fall, pituitary surgery, delivery, leuprolide injection, triple bolus test, anticoagulative and antithrombotic therapy, and pituitary function test $[6,9,12,13,15,26,31,37]$.

To confirm the diagnosis of cerebral infarction caused by PA, clinical symptoms plus brain CT or MRI findings are required. During the early disease phase, cranial MRI is more useful than CT. Therefore, when PA is clinically suspected, cranial MRI should be performed immediately $[19,26]$. Angiography, including CT angiography and MR angiography, can be used to detect changes in blood vessels, including damaged vascular areas and impaired blood vessels affected by compression or paralysis, as well as the patency of intracranial blood vessels and anatomical relationships between blood vessels and tumours; the identification of these factors can help elucidate the pathophysiological mechanism of the disease and determine the appropriate timing for early tumour decompression [17].

Cerebral infarction caused by PA usually begins with sudden neurological symptoms. The usual symptoms are cephalalgia, visual impairment, and extraocular muscle paralysis followed by disturbance of consciousness, limb weakness, and hemiplegia. Because cerebral infarction caused by PA involves the bilateral ICA, ACA, and MCA; other large intracranial blood vessels; and multiple branches of these blood vessels, the clinical symptoms are generally serious, and the mortality rate is high. In this review, the mortality rate was as high as $26.8 \%$. One patient was in a vegetative state, and more than half had disturbance of consciousness; these clinical manifestations are far more serious than those of general cerebral infarction. Most surviving patients had severe disability, such as hemiplegia, aphasia, and visual impairment. However, the mortality of patients with PA alone ranges from 0 to 12.5, which shows that patients with PA alone have a better prognosis than patients with cerebral infarction caused by PA $[45,46]$. The cause of death is usually a combination of cerebral infarction and oedema in these cases $[7,14,19]$.

There are 2 main pathophysiological mechanisms of the disease: one is the compression of intracranial blood vessels by a tumour and the other is blood vessel spasm caused by tumour bleeding [2]. In 25 patients with cerebral infarction caused by PA, infarction was due to direct intracranial vascular compression $[4-6,10,14,19,21$, 23-26, 29, 30, 33-35, 37-39, 41-44]. The tumour constantly grows and then presses on the adjacent MCA and ACA, causing the corresponding symptoms and signs. The ACA is more prone to compression than MCA. When the tumour expands to the cavernous sinus on both sides, it can cause paralysis of the extraocular muscles and compression of the ICA. In general, tumours compress unilateral arteries, but 5 cases of bilateral ICA compression $[7,9,19,26,29]$ and 3 cases of bilateral ACA compression have been reported in the literature $[18,28$, 39]. Cerebral vasospasm is another mechanism of cerebral infarction caused by PA $[2,7,8,11-13,15,17,18,21$, $22,27,28,31,32,40]$. First, pituitary tumour haemorrhage enters the subarachnoid space. Then, the pituitary tumour secretes vasoactive substances. Finally, the compressed hypothalamus releases vasoactive substances. In severe cases, multiple causes coexist in PA and lead to cerebral infarction. Two studies have reported that the mechanism of cerebral infarction is vascular compression and spasm $[16,36]$. If patients do not have an angiogram, it is not clear whether vasospasm or intracranial arterial compression occurs when pituitary stroke causes cerebral infarction.

Whether cerebral infarction caused by PA requires early surgery to relieve compression or restore the blood supply is controversial. In some cases, tumour decom- 
pression can restore blood flow to the compressed vessels and reduce intratumoural stress, thereby reducing the exudation of blood or vasoactive substances into the subarachnoid space; thus, some neurological deficits can be resolved $[2,30]$. However, some authors have reported that surgical decompression might be detrimental to patient recovery if a head CT indicates cerebral infarction and might result in haemorrhagic infarction $[10,30,47]$. In our study, compared to patients who underwent emergency surgery, patients who underwent delayed surgery showed a better prognosis. There are 2 possible reasons for this observation: one reason is that most patients who underwent early emergency surgery were in a critical state of consciousness (Table 1) and the other reason is that all patients who underwent delayed surgery survived, which may be related to the general stability of the patients after hormone replacement therapy and symptomatic supportive treatment. Compared to patients who underwent craniotomy, patients who underwent TSS not only improved well but also had a lower mortality rate (Table 3 ). The reason for this result might be that the approach of TSS for the treatment of pituitary adenoma not only provides a good surgical field but can also reduce trauma and avoid brain tissue damage $[2,48]$.

The symptoms of ischaemic stroke and cerebral infarction caused by PA are similar and include limb hemiplegia, speech difficulty, and facial paralysis, and both conditions are characterized by a transient ischaemic attack. Thus, it is sometimes difficult to distinguish between these 2 diseases. However, the treatments for the 2 diseases are different. For general ischaemic stroke, common treatment methods include anticoagulant or antiplatelet drugs and thrombolytic therapy, while for cerebral infarction caused by PA, alternatively, perhaps "TSS, craniotomy decompression, and hormone replacement therapy" would also work well. If a cerebral infarction caused by PA is treated as a general cerebral infarction, it will aggravate the patient's condition. Roberto reported a case in which the patient presented with a transient ischaemic attack that was aggravated by antiplatelet therapy [35]. Similarly, in this article, treatment with antiplatelet and anticoagulant drugs also induced cerebral infarction caused by PA and aggravated the condition [26]. Therefore, it is very important to identify the two diseases correctly.

\section{Limitations}

Because cerebral infarction caused by PA is uncommon, only a small number of cases have been reported thus far. It is inevitable to have retrospective bias when only the cases of others are reviewed and when the number of cases is small. Therefore, the findings of this analysis are not sufficient to demonstrate which treatment is best for cerebral infarction caused by PA, and it is necessary for more studies to be carried out to explore the disease.

\section{Conclusions}

In conclusion, cerebral infarction caused by PA is a rare and critical condition, and the treatment for this type of infarction is different from the treatment for general acute cerebral infarction. Transsphenoidal tumour decompression causes relatively little trauma to the patient and has a low mortality rate. Therefore, TSS is a suitable treatment recommendation. Furthermore, we hope that more research will be conducted to explore the disease and find better methods for diagnosis and treatment.

\section{Conflict of Interest Statement}

The authors have no conflicts of interest.

\section{Funding Sources}

This study was supported by grants from the National Natural Science Foundation of China (81671134), the Southern China International Cooperation Base for Early Intervention and Functional Rehabilitation of Neurological Diseases (2015B050501003), Guangdong Provincial Engineering Center for Major Neurological Disease Treatment, Guangdong Provincial Translational Medicine Innovation Platform for Diagnosis and Treatment of Major Neurological Disease, and Guangdong Provincial Clinical Research Center for Neurological Diseases.

\section{Author Contributions}

J.Q.H. and X.S. were responsible for the design, literature search, discussion, and first draft. H.H. offered help in the design, writing, and critical comments. S.L.M. and H.X.Y. were responsible for the literature search and discussion. C.X.H. helped in the literature search. 


\section{References}

1 Kim JP, Park BJ, Kim SB, Lim YJ. Pituitary apoplexy due to pituitary adenoma infarction. J Korean Neurosurg Soc. 2008;43(5):246-9.

2 Zou Z, Liu C, Sun B, Chen C, Xiong W, Che $\mathrm{C}$, et al. Surgical treatment of pituitary apoplexy in association with hemispheric infarction. J Clin Neurosci. 2015;22(10):1550-4.

3 Abbas MS, AlBerawi MN, Al Bozom I, Shaikh NF, Salem KY. Unusual complication of pituitary macroadenoma: a case report and review. Am J Case Rep. 2016;17:707-11.

4 Schnitker MT, Lehnert HB. Apoplexy in a pituitary chromophobe adenoma producing the syndrome of middle cerebral artery thrombosis; case report. J Neurosurg. 1952; 9(2):210-3.

5 Sakalas R, David RB, Vines FS, Becker DP. Pituitary apoplexy in a child. Case report. J Neurosurg. 1973;39(4):519-22.

6 Rosenbaum TJ, Houser OW, Laws ER. Pituitary apoplexy producing internal carotid artery occlusion. Case report. J Neurosurg. 1977;47(4):599-604.

7 Cardoso ER, Peterson EW. Pituitary apoplexy and vasospasm. Surg Neurol. 1983;20(5):3915.

8 Majchrzak H, Wencel T, Dragan T, Bialas J. Acute hemorrhage into pituitary adenoma with $\mathrm{SAH}$ and anterior cerebral artery occlusion. Case report. J Neurosurg. 1983; 58(5):771-3.

9 Bernstein M, Hegele RA, Gentili F, Brothers M, Holgate R, Sturtridge WC, et al . Pituitary apoplexy associated with a triple bolus test. Case report. J Neurosurg. 1984;61(3):586-90.

10 Clark JD, Freer CE, Wheatley T. Pituitary apoplexy: an unusual cause of stroke. Clin Radiol. 1987;38(1):75-7.

11 Pozzati E, Frank G, Nasi MT, Giuliani G. Pituitary apoplexy, bilateral carotid vasospasm, and cerebral infarction in a 15 -year-old boy. Neurosurgery. 1987;20(1):56-9.

12 Itoyama Y, Goto S, Miura M, Kuratsu J, Ushio $\mathrm{Y}$, Matsumoto T. Intracranial arterial vasospasm associated with pituitary apoplexy after head trauma: case report. Neurol Med Chir. 1990;30(5):350-3.

13 Ludecke D. Subarachnoid haemorrhage and vasospasm due to pituitary apoplexy after pituitary function tests: comment. Acta Neurochirurgica. 1999;141(9):1010.

14 Lath R, Rajshekhar V. Massive cerebral infarction as a feature of pituitary apoplexy. Neurol India. 2001;49(2):191-3.

15 Engel G, Huston M, Oshima S, Beck C, Harsh $\mathrm{G}$, Rosenthal MH, et al. Pituitary apoplexy after leuprolide injection for ovum donation. J Adolesc Health. 2003;32(1):89-3.

16 Rodier G, Mootien Y, Battaglia F, Martinet O, Cohen E. Bilateral stroke secondary to pituitary apoplexy. J Neurol. 2003;250(4):494-5.

17 Akutsu H, Noguchi S, Tsunoda T, Sasaki M, Matsumura A. Cerebral infarction following pituitary apoplexy: case report. Neurol Med Chir. 2004;44(9):479-83.
18 Bhansali A, Dutta P, Khandelwal N, Pathak A, Vashisht R. Pituitary apoplexy: an unusual cause of frontal lobe syndrome. Australas $\mathrm{Ra}$ diol. 2005;49(2):127-31.

19 Kurschel S, Leber KA, Scarpatetti M, Roll P. Rare fatal vascular complication of transsphenoidal surgery. Acta Neurochir. 2005; 147(3):321-5; discussion 5.

20 Jeon BC, Park YS, Oh HS, Kim YS, Chun BK. Pituitary apoplexy complicated by chemical meningitis and cerebral infarction. J Korean Med Sci. 2007;22(6):1085-9.

21 Ahmed SK, Semple PL. Cerebral ischaemia in pituitary apoplexy. Acta Neurochir. 2008; 150(11):1193-6; discussion 6.

22 Ben-Nakhi A, Muttikkal TJ, Chavan VN, AlTurkomani AY, Gupta R. Pituitary apoplexy: a rare cause of cerebral infarction. A case report. Neuroradiol J. 2008;21(5):661-5.

23 Das NK, Behari S, Banerji D. Pituitary apoplexy associated with acute cerebral infarct. J Clin Neurosci. 2008;15(12):1418-20.

24 Dogan S, Kocaeli H, Abas F, Korfali E. Pituitary apoplexy as a cause of internal carotid artery occlusion. J Clin Neurosci. 2008; 15(4):480-3.

25 Yang SH, Lee KS, Lee KY, Lee SW, Hong YK. Pituitary apoplexy producing internal carotid artery compression: a case report. J Korean Med Sci. 2008;23(6):1113-7.

26 Lill CM, Hoch H, Dieste FJ, Vogel HP, Zipp F, Paul F. Bilateral stroke following pituitary apoplexy. J Clin Neurosci. 2009;16(12):1670-

27 Cerase A, Tarantino A, Muzii VF, Vittori C, Venturi C. Vasospasm and cerebral infarction from pituitary apoplexy. A case report. Neuroradiol J. 2010;23(3):321-4.

28 Mohindra S, Kovai P, Chhabra R. Fatal bilateral ACA territory infarcts after pituitary apoplexy: a case report and literature review. Skull Base. 2010;20(4):285-8.

29 Perotti V, Dexter M. Post-partum pituitary apoplexy with bilateral third nerve palsy and bilateral carotid occlusion. J Clin Neurosci. 2010;17(10):1328-30.

30 Chokyu I, Tsuyuguchi N, Goto T, Chokyu K, Chokyu M, Ohata K. Pituitary apoplexy causing internal carotid artery occlusion: case report. Neurol Med Chir. 2011;51(1):48-51.

31 Gupta R, Sharma A, Vaishya R, Tandon M. Ischemic complications after pituitary surgery: a report of two cases. J Neurol Surg A Cent Eur Neurosurg. 2013;74(Suppl 1):e119_ 23.

32 Radhiana H, Syazarina SO, Shahizon Azura AM, Azizi AB. Pituitary apoplexy: a rare cause of middle cerebral artery infarction. Med J Malaysia. 2013;68(3):264-6.

33 Navarro-Bonnet J, Martínez-Anda JJ, Balderrama-Soto A, Pérez-Reyes SP, Pérez-Neri I, Portocarrero-Ortiz L. Stroke associated with pituitary apoplexy in a giant prolactinoma: a case report. Clin Neurol Neurosurg. 2014; 116:101-3.
34 Rebeiz T, Cueva W, Ardelt A. Unusual case of bilateral caudate infarcts following pituitary apoplexy. JAMA Neurol. 2014;71(2):226-7.

35 Rey-Dios R, Payner TD, Cohen-Gadol AA. Pituitary macroadenoma causing symptomatic internal carotid artery compression: surgical treatment through transsphenoidal tumor resection. J Clin Neurosci. 2014;21(4): 541-6.

36 Zhang C, Feng F, Zhu Y, Wang R, Xing B. Cerebral infarction caused by pituitary apoplexy: case report and review of literature. Turk Neurosurg. 2014;24(5):782-7.

37 Banerjee C, Snelling B, Hanft S, Komotar RJ Bilateral cerebral infarction in the setting of pituitary apoplexy: a case presentation and literature review. Pituitary. 2015;18(3):352-8.

38 Kasl RA, Hughes J, Burrows AM, Meyer FB. Pediatric ischemic stroke from an apoplectic prolactinoma. Childs Nerv Syst. 2015; 31(8):1387-92.

39 Abbas MS, AlBerawi MN, Al Bozom I, Shaikh NF, Salem KY. Unusual complication of pituitary macroadenoma: a case report and review. Am J Case Rep. 2016;17:707-11.

40 Gambaracci G, Rondoni V, Guercini G, Floridi P. Pituitary apoplexy complicated by vasospasm and bilateral cerebral infarction. BMJ Case Rep. 2016;2016:bcr2016216186.

41 Sussman ES, Ho AL, Pendharkar AV, Achrol AS, Harsh GR. Pituitary apoplexy associated with carotid compression and a large ischemic penumbra. World Neurosurg. 2016; 92:581-e13.

42 Pasha SA, Ranganthan LN, Setty VK, Reddy $\mathrm{R}$, Ponnuru DA. Acute ischaemic stroke as a manifestation of pituitary apoplexy in a young lady. J Clin Diagn Res. 2017; 11(5):OD03-OD5.

43 Bettag C, Strasilla C, Steinbrecher A, Gerlach R. Unilateral tuberothalamic artery ischemia caused by pituitary apoplexy. J Neurol Surg A Cent Eur Neurosurg. 2018;79(6):550-4.

44 Nagure PV, Nikam VR, Garud AS. Pituitary apoplexy producing internal carotid and basilar artery compression: a rare case report. Asian J Neurosurg. 2018;13(4):1264-8.

45 Bujawansa S, Thondam SK, Steele C, Cuthbertson DJ, Gilkes CE, Noonan C, et al. Presentation, management and outcomes in acute pituitary apoplexy: a large single-centre experience from the United Kingdom. Clin Endocrinol. 2014;80(3):419-24.

46 Dubuisson AS, Beckers A, Stevenaert A. Classical pituitary tumour apoplexy: clinical features, management and outcomes in a series of 24 patients. Clin Neurol Neurosurg. 2007; 109(1):63-70

47 Han F, Peng B, Gao S, Mao CH, Cui LY, Xing $\mathrm{B}$, et al. Clinical reasoning: a 42-year-old man with severe headache, fever, and acute coma. Neurology, 2014:82(2):e9-13.

48 Solari D, Cavallo LM, Cappabianca P. Surgical approach to pituitary tumors. Handb Clin Neurol. 2014;124:291-301. 